\title{
Assessing Genetic Diversity in Dual Purpose Oat (Avena sativa L.) Cultivars Based on Morphological and Quality Traits
}

\author{
Rajvir Kaur', Rahul Kapoor ${ }^{1 *}$, Yogesh Vikal ${ }^{2}$ and Kamaljit Kaur ${ }^{3}$ \\ ${ }^{1}$ Department of Plant Breeding and Genetics, ${ }^{2}$ School of Agricultural Biotechnology, \\ ${ }^{3}$ Department of Biochemistry, Punjab Agricultural University, Ludhiana, Punjab, India \\ *Corresponding author
}

\begin{tabular}{|l|}
\hline Ke y w o r d s \\
Oat (Avena sativa \\
$\begin{array}{l}\text { L.), Genetic } \\
\text { Diversity, Quality } \\
\text { Traits }\end{array}$ \\
\hline Article Info \\
\hline $\begin{array}{l}\text { Accepted: } \\
\text { 12 April } 2018 \\
\text { Available Online: } \\
\text { 10 May } 2018\end{array}$ \\
\hline
\end{tabular}

\section{Introduction}

Oat is regarded as most important cereal crop throughout the world and used as an important source of essential nutrients for human consumption (Boczkoswka and Tarczyk, 2013). Oat (Avena sativa L.) is a highly

\section{A B S T R A C T}

The present study was undertaken to assess the genetic diversity present in the ninety six oat (Avena sativa L.) germplasm lines representing the collection from various ecogeographical regions of the country. On the basis of mean performance of the genotypes for fodder traits; OL 10 for plant height $(60.09 \mathrm{~cm})$, OL 1636 for leaf length $(53.55 \mathrm{~cm})$ and JO 03-95 for leaf width $(1.86 \mathrm{~cm})$, OS 7 for GFY $(3.38 \mathrm{~kg} / \mathrm{plot})$ and JHO-2001-1 for DMY $(0.61 \mathrm{~kg} / \mathrm{plot})$ were found to be superior. Similarly, on basis of mean performance of the genotypes for grain traits; OS 7 for beta-glucan (4.35\%), JHO-2009-1 for grain length (15 $\mathrm{mm}$ ), OL 1542, OL 1611, OL 1615, OL 1636, OL 1635, JHO 851, EC 605839, EC 605833, EC 209750, EC 209472, EC 209408, SKO 315, SKO 312, and RO-2001-1 for grain width $(3.3 \mathrm{~mm})$ and UPO-093 for grain yield $(426.91 \mathrm{~g} / \mathrm{plot})$ were found to be superior. Genetic divergence among 96 accessions was worked out for fodder and grain traits and then for dual purpose to generate dendrogram based on complete linkage and squared euclidean distance. All the 96 accessions were grouped into 6 clusters. Maximum inter cluster distance for fodder, grain and dual purpose was recorded between clusters VI and III (9.99), clusters I and VI (9.06), clusters IV and V (11.24) respectively, suggesting significant high genetic diversity between genotypes of these clusters. According to criteria followed by Proceedings of AICRP (FCU 2015), the 14 best dual purpose genotypes evaluated are: UPO 093, OL 1611, JHO-2001-1, HJ 114, JHO 851, OL 1635, OS 329, SKO 27, HJ 8, OS 363, OL 1714, OS 376, EC 605833 and JHO-2009-1. 
one of the best dual purpose cereal crops that fit well into the platter of human and cattle as well. For oats to classify as a dual purpose crop, it should have high green fodder and grain yield harvested from the same crop where the first cut is taken for fodder and subsequently the crop is harvested at the time of grain maturity.

Increased oat consumption is often enhanced due to nutritional attributes including antioxidants and high soluble fiber (Rasane et al., 2015). Oats is good source of antioxidants like avenanthramides, alpha- tocopherol, alpha-tocotrienol and also total dietary fiber including beta-glucans (Oliver et al., 2010). Latest research have analyzed the oat consumption effects on health and benefits on health are beyond reducing cardio vascular risk like diabetes, controls blood-pressure levels, lowers blood cholesterol concentrations, controls and maintains weight and gastro-intestinal health (Clemens, 2014).

Morphological evaluation of a germplasm collection is useful to describe its genetic diversity and to identify agronomically significant variation. Such an assessment involves characterization of variations for various morphological traits.

Before embarking on a breeding programme, assessment of germplasm collection for key agronomic traits, seed quality and defensive traits, flowering, maturity, plant height, protein content, oil content, primary branches, number of capsules, resistances to pests and diseases, drought and cold tolerances and other worthwhile traits is important (Krull and Berlaug, 1970).

Morphological traits are associated with a relatively small number of loci, thus the potential difference could be lost in the analysis of large amounts of molecular data (Diederichsen, 2009).

\section{Materials and Methods}

\section{Plant material}

The experimental material consists of 96 genotypes from diverse eco-geographic regions of the country maintained at the experimental area of Forage Research Farm, Department of Plant Breeding and Genetics, Punjab Agricultural University, Ludhiana. The names and origin of the plant material used in the present investigation has been given in Table 1. Ninety-six Avena sativa accessions were morphologically analysed by Augmented Design. Each entry was accommodated in two rows of $2 \mathrm{~m}$ length and three checks viz., OL 125 (zonal check), OL-10 (state check) and Kent (national check) were repeated randomly among each block to obtain an estimate of the error. To raise the healthy crop, recommended agronomic practices were followed. Oat germplasm lines were analysed during Rabi 2014-15 for various morpho- agronomic traits.

\section{Morphological analysis}

Data on five randomly tagged plants of each entry per genotype was recorded. Data for some of the morpho-agronomic traits viz; days to flowering, days to maturity, green fodder yield, dry matter yield, stover yield were taken on the plot basis and rest of the data for other characters were recorded from tagged plants only. A total of 34 morphological traits viz., PH 1-Plant Height in first cut, NOL/plant -No. of Leaves per Plant, LL 1-Leaf Length in first cut, LW 1-Leaf Width in first cut, NOT-No. of Tillers per Plant, SG 1-Stem Girth in first cut, GFY (kg/plot)-Green Fodder Yield, DMY (kg/plot)-Dry Matter Yield, RG \%Regeneration Percentage, LSR-Leaf Stem Ratio, DF-Days to Flowering, DM-Days to Maturity, LAI-Leaf Area Index, ADF \%-Acid Detergent Fibre (\%), NDF\%-Neutral Detergent Fibre (\%), IVDMD\%-In-Vitro Dry Matter Digestibility Percentage, CP\%-Crude 
Protein, $\beta$ - G\%- beta-glucan, PL-Panicle Length, GL-Grain Length, GW-Grain Width, 1000 GW-Thousand Grain Weight, SY-Stover Yield, GY-Grain Yield, NOET per meter rowNo. of Effective Tillers per Meter Row Length, SNPP-Spikelet No. per Panicle, FNPP-Floret No. per Panicle, GNPP-Grain No. per Panicle, PH 2-Plant Height in second cut, FLL-Flag Leaf Length, FLW-Flag Leaf Width, LL2-Leaf Length in second cut, LW 2Leaf Width in second cut, SG 2-Stem Girth in second cut.

\section{Results and Discussion}

\section{Analysis of variance (ANOVA)}

The analysis was carried out using the software SPAD (Rahore et al., 2004). Data with respect to check varieties were subjected to analysis of variance as per Augmented design (Federer, 1956) to obtain adjusted trait values for 3 checks as well as for 93 test genotypes.

\section{Analysis of genetic divergence}

The analysis of genetic divergence was done by using Minitab software (Barbara 1972). Cluster analysis is a multivariate technique which aims to classify a sample on basis of a set of measured variables into a number of different groups such that similar subjects are placed in same group. It provides a way for scientists to discover potential relationships and assists to construct systematic structures in large number of variables and observations. Clustering methods includes analysis of characterizing data on genotypes from which the core collection is to be selected (Hintum and Knupffer, 1995).

The analyses of variances (Table 2) were carried out using data recorded for morphoagronomic traits on three check varieties and 93 test genotypes sown in augmented block design for dual purpose during Rabi 2014-15. The mean square for blocks (adjusted) were significant for ADF\% for fodder and 1000 GW, GY, NOET per meter row, FLL, FLW, GNPP, FNPP for grain related traits indicating the low heterogeneity among the blocks. The mean square for treatments (Adjusted) were found significant for PH 1, LL 1, LW 1, GFY, DMY, RG\%, DM, DF, LAI, ADF, CP for fodder and beta glucan, PL, GL, GY, SY, 1000 GW, SNPP, FLL, FLW, SG, LL 2 indicating that the significant differences were present between the genotypes for these traits. Contrast analysis was computed to examine the experimental material in terms of variation present among the checks (controls), among the test genotypes (treatments) and test genotypes $v s$ checks. The mean square for $\mathrm{PH}$ 1 (cm), GFY (kg/plot), DMY (kg/plot), DF was significant among checks for fodder traits and beta glucan, GY, SNPP for grain traits, revealing that the significant differences were present between the three checks. Among the test genotypes most of the traits viz; $\mathrm{PH} 1$ (cm), LW 1 (cm), RG\%, DF, DM, LAI, $\mathrm{ADF} \%, \mathrm{CP} \%$ for fodder and 1000GW, SY, NOET per meter row for grain traits showed significant mean square values, indicating differences among the test genotypes for these traits. PH $1(\mathrm{~cm}), \mathrm{LW} 1(\mathrm{~cm})$, GFY (kg/plot), DMY (kg/plot), LSR, DF, DM, LAI and CP\% for fodder and beta glucan, PL, 1000GW, GY, SNPP, FLL, FLW, LW 2 and SG 2 for grain showed significant mean square suggesting significant differences for test genotypes $v s$ controls for these traits.

\section{Distribution of accessions into different clusters}

Genetic divergence among 96 accessions (93 test genotypes and 3 checks) of oats was worked out for fodder and grain traits and then for dual data, to generate dendrogram based on complete linkage, Squared Euclidean Distance (Fig. 1-3). 
Fig.1 Dendrogram for fodder traits

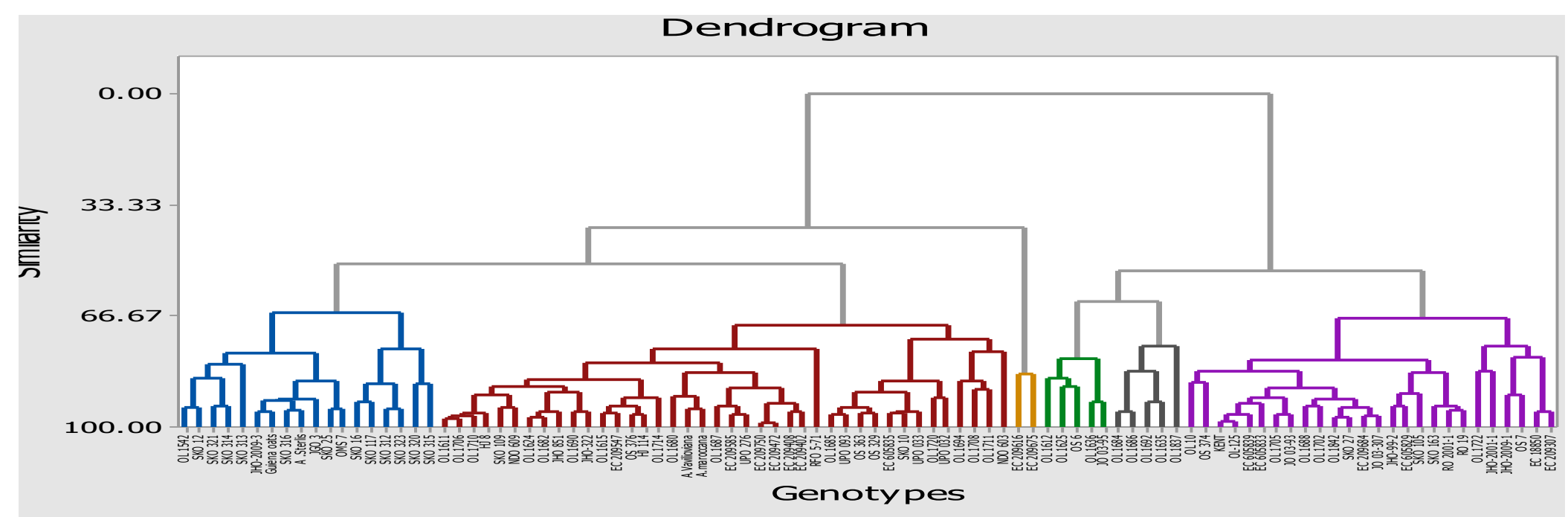

Fig.2 Dendrogram for grain traits

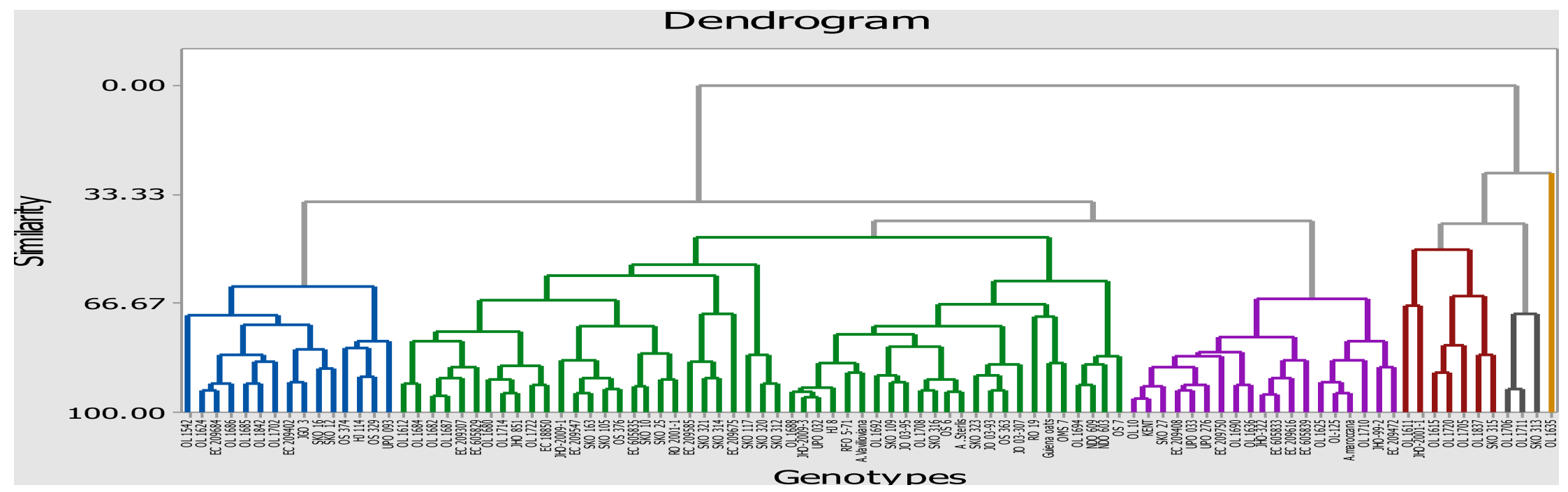


Fig.3 Dendrogram for dual purpose

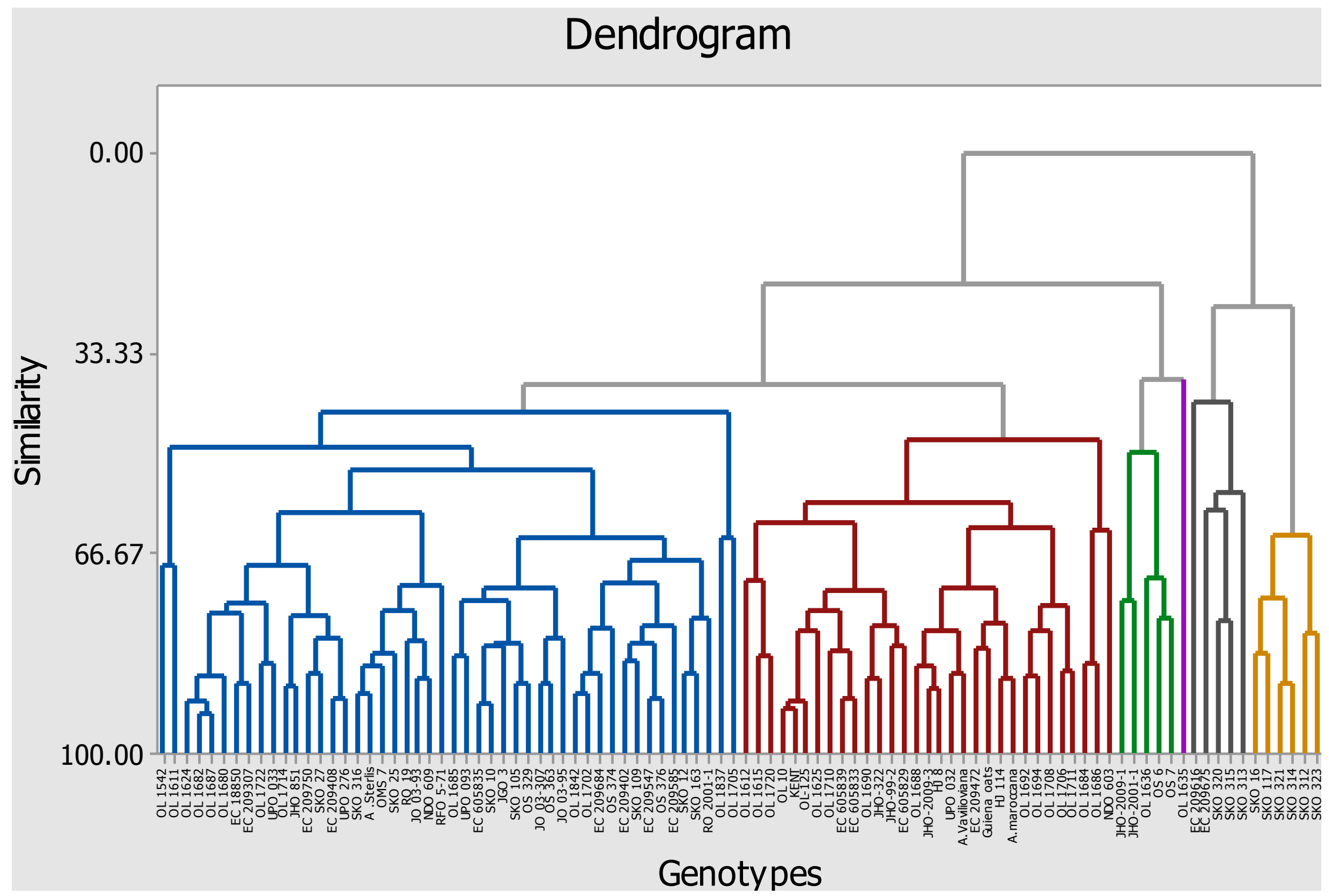


Table.1 List of source germplasm evaluated in present study

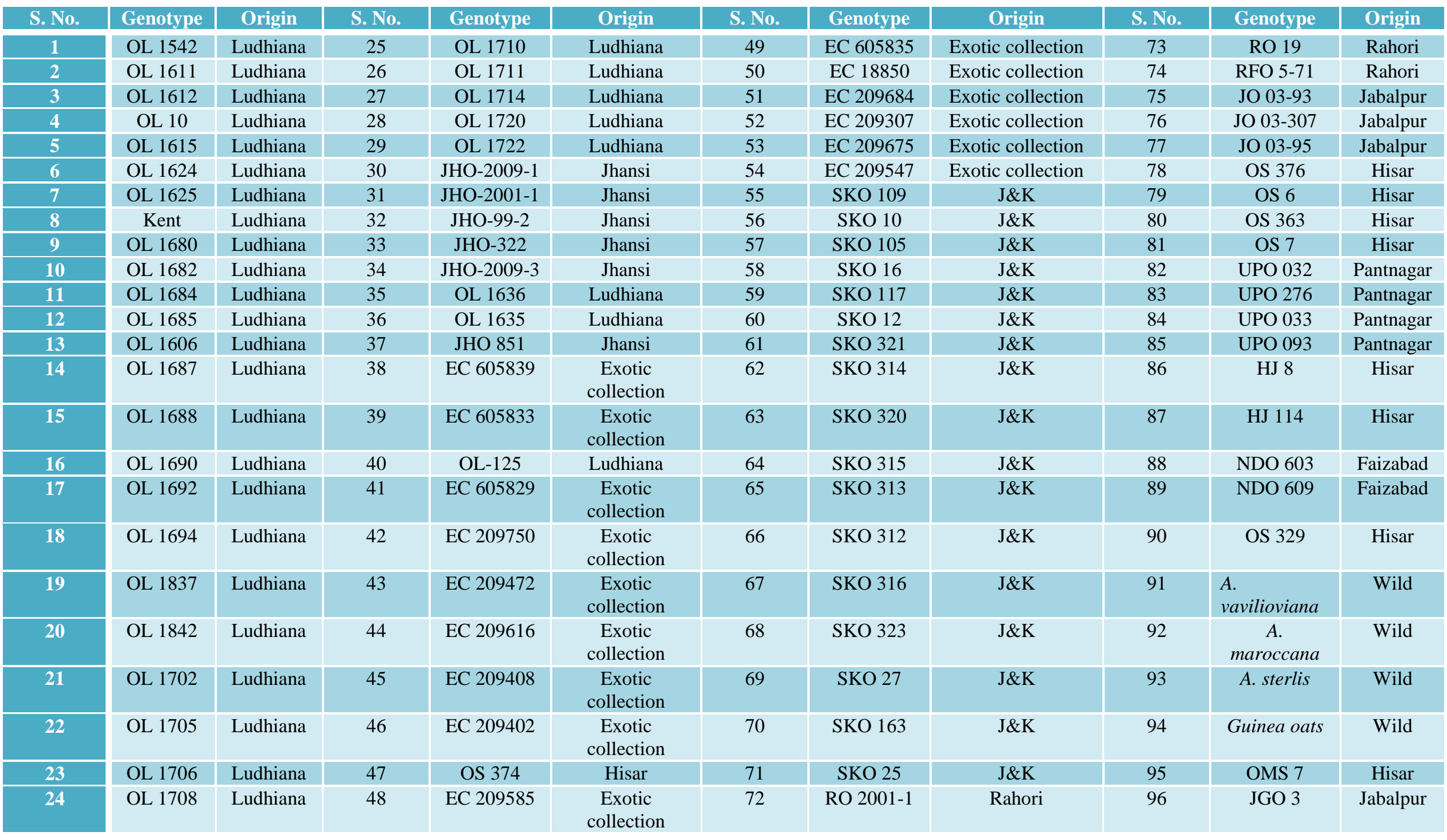


Table.2 Analysis of variance (ANOVA) for the morpho-agronomic traits in oats

For Fodder

\begin{tabular}{|c|c|c|c|c|c|c|c|c|c|c|}
\hline Source & d.f. & PH 1 (cm) & NOL/plant & LL 1(cm) & LW 1(cm) & NOT & $\begin{array}{l}\text { SG } 1 \\
(\mathrm{~mm})\end{array}$ & GFY(kg/plot) & DMY(kg/plot) & RG\% \\
\hline Block (Adj.) & 10 & $3.85(0.5830)$ & $\begin{array}{c}131.29 \\
(0.457006)\end{array}$ & $\begin{array}{c}1.53 \\
(0.999529)\end{array}$ & $\begin{array}{c}0.01 \\
(0.361819)\end{array}$ & $\begin{array}{c}6.48 \\
(0.457006)\end{array}$ & $\begin{array}{c}0.26 \\
(0.862048)\end{array}$ & $\begin{array}{c}0.05 \\
(0.718466)\end{array}$ & $\begin{array}{c}0.005 \\
(0.345153)\end{array}$ & $\begin{array}{l}48.03 \\
(0.103707)\end{array}$ \\
\hline $\begin{array}{l}\text { Treatments } \\
\text { (Adj.) }\end{array}$ & 95 & $\begin{array}{c}26.92 \\
(0.000018)^{* *}\end{array}$ & $\begin{array}{c}207.54 \\
(0.108101)\end{array}$ & $\begin{array}{c}40.94 \\
(0.004459) * *\end{array}$ & $\begin{array}{c}0.02 \\
(0.007778)^{* *}\end{array}$ & $\begin{array}{c}10.25 \\
(0.108101)\end{array}$ & $\begin{array}{c}7.63 \\
(0.143081)\end{array}$ & $\begin{array}{c}0.16 \\
(0.039158)^{*}\end{array}$ & $\begin{array}{c}0.008 \\
(0.030309)^{*}\end{array}$ & $\begin{array}{c}74.42 \\
(0.003663)^{*}\end{array}$ \\
\hline Error & 20 & 4.48 & 127.93 & 14.21 & 0.008 & 6.32 & 0.50 & 0.084 & 0.004 & 25.07 \\
\hline \multicolumn{11}{|c|}{ Contrast Analysis } \\
\hline \multirow{3}{*}{$\begin{array}{l}\text { (i) Among } \\
\text { control } \\
\text { (ii) Among } \\
\text { test } \\
\text { genotypes } \\
\text { (iii) Test-vs } \\
\text { control }\end{array}$} & 2 & $\begin{array}{c}225.86 \\
(0.000010)^{* *}\end{array}$ & $\begin{array}{c}329.52 \\
(0.101076)\end{array}$ & $\begin{array}{c}41.98 \\
(0.075115)\end{array}$ & $\begin{array}{c}0.01 \\
(0.179735)\end{array}$ & $\begin{array}{c}16.27 \\
(0.101076)\end{array}$ & $\begin{array}{c}0.61 \\
(0.315170)\end{array}$ & $\begin{array}{c}0.35 \\
(0.031226) *\end{array}$ & $\begin{array}{c}0.02 \\
(0.013130)^{*}\end{array}$ & $\begin{array}{c}15.90 \\
(0.540568)\end{array}$ \\
\hline & 92 & $\begin{array}{c}9.33 \\
(0.031982)^{*}\end{array}$ & $\begin{array}{c}205.72 \\
(0.112719)\end{array}$ & $\begin{array}{c}38.36 \\
(0.006845)\end{array}$ & $\begin{array}{c}0.01 \\
(0.023351)^{*}\end{array}$ & $\begin{array}{c}10.16 \\
(0.112719)\end{array}$ & $\begin{array}{c}0.74 \\
(0.157136)\end{array}$ & $\begin{array}{c}0.12 \\
(0.156725)\end{array}$ & $\begin{array}{c}0.006 \\
(0.119525)\end{array}$ & $\begin{array}{c}75.45 \\
(0.003377)^{* *}\end{array}$ \\
\hline & 1 & $\begin{array}{c}1283.99 \\
(0.000010)^{* *}\end{array}$ & $\begin{array}{c}121.48 \\
(0.341461)\end{array}$ & $\begin{array}{c}279.43 \\
(0.000255)\end{array}$ & $\begin{array}{c}0.39 \\
(0.000010)^{* *}\end{array}$ & $\begin{array}{c}5.99 \\
(0.341461)\end{array}$ & $\begin{array}{c}2.69 \\
(0.031169)\end{array}$ & $\begin{array}{c}3.89 \\
(0.000010)^{* *}\end{array}$ & $\begin{array}{c}0.18 \\
(0.000010)^{* *}\end{array}$ & $\begin{array}{c}76.45 \\
(0.096130)\end{array}$ \\
\hline
\end{tabular}

\begin{tabular}{|c|c|c|c|c|c|c|c|c|c|}
\hline Source & d.f. & LSR & DF & DM & LAI & ADF \% & NDF \% & IVDMD\% & СР\% \\
\hline Block (Adj.) & 10 & $\begin{array}{c}0.01 \\
(0.091557)\end{array}$ & $\begin{array}{c}13.42 \\
(0.562430)\end{array}$ & $\begin{array}{c}19.74 \\
(0.452105)\end{array}$ & $\begin{array}{c}3.19 \\
(0.352500)\end{array}$ & $\begin{array}{c}39.82 \\
(0.000550)^{* *}\end{array}$ & $\begin{array}{c}43.98 \\
(0.709081)\end{array}$ & $3.97(0.4631)$ & $\begin{array}{l}0.33 \\
(0.867649)\end{array}$ \\
\hline $\begin{array}{l}\text { Treatments } \\
\text { (Adj.) }\end{array}$ & 95 & $\begin{array}{c}0.01 \\
(0.612544)\end{array}$ & $\begin{array}{c}37.48 \\
(0.011797)^{*}\end{array}$ & $\begin{array}{c}37.89 \\
(0.040851)^{*}\end{array}$ & $\begin{array}{c}10.86 \\
(0.000409)^{* *}\end{array}$ & $\begin{array}{c}15.80 \\
(0.022389)^{*}\end{array}$ & $\begin{array}{c}46.77 \\
(0.822612)\end{array}$ & $\begin{array}{c}1.79 \\
(0.972121)\end{array}$ & $\begin{array}{c}1.62 \\
(0.012715)^{*}\end{array}$ \\
\hline Error & 20 & 0.01 & 15.18 & 19.10 & 2.68 & 7.13 & 62.32 & 3.75 & 0.664936 \\
\hline \multicolumn{10}{|c|}{ Contrast Analysis } \\
\hline \multirow{3}{*}{$\begin{array}{l}\text { (i) Among } \\
\text { control } \\
\text { (ii) Among test } \\
\text { genotypes } \\
\text { (iii) Test vs } \\
\text { control }\end{array}$} & 2 & $\begin{array}{c}0.01 \\
(0.491179)\end{array}$ & $\begin{array}{c}78.56 \\
(0.015431)^{*}\end{array}$ & $\begin{array}{c}15.64 \\
(0.455318)\end{array}$ & $\begin{array}{c}0.21 \\
(0.921790)\end{array}$ & $\begin{array}{c}4.10 \\
(0.571664)\end{array}$ & $\begin{array}{c}8.34 \\
(0.875475)\end{array}$ & $\begin{array}{c}0.29 \\
(0.783601)\end{array}$ & $\begin{array}{c}1.96 \\
(0.075094)\end{array}$ \\
\hline & 92 & $\begin{array}{c}0.01 \\
(0.706931)\end{array}$ & $\begin{array}{c}35.99 \\
(0.015155)^{*}\end{array}$ & $\begin{array}{c}37.59 \\
(0.042858)^{*}\end{array}$ & $\begin{array}{c}8.54 \\
(0.002315)^{* *}\end{array}$ & $\begin{array}{c}16.22 \\
(0.019465)^{*}\end{array}$ & $\begin{array}{c}47.58 \\
(0.807760)\end{array}$ & $\begin{array}{c}1.82 \\
(0.969016)\end{array}$ & $\begin{array}{c}1.60 \\
(0.013833)^{*}\end{array}$ \\
\hline & 1 & $\begin{array}{c}0.046 \\
(0.008258)^{* *}\end{array}$ & $\begin{array}{c}95.50 \\
(0.020852)^{*}\end{array}$ & $\begin{array}{c}113.35 \\
(0.024329)^{*}\end{array}$ & $\begin{array}{c}255.27 \\
(0.000010)^{* *}\end{array}$ & $\begin{array}{c}0.33 \\
(0.831756)\end{array}$ & $\begin{array}{c}52.43 \\
(0.369965)\end{array}$ & $\begin{array}{c}0.45 \\
(0.736104)\end{array}$ & $\begin{array}{c}2.94 \\
(0.048121)^{*}\end{array}$ \\
\hline
\end{tabular}


For Grain

\begin{tabular}{|c|c|c|c|c|c|c|c|c|c|c|}
\hline Source & df & $\beta-G \%$ & PL & GL & GW & $1000 \mathrm{GW}$ & SY & GY & $\begin{array}{c}\text { NOET/m } \\
\text { Row }\end{array}$ & SNPP \\
\hline $\begin{array}{l}\text { Block } \\
\text { (Adj.) } \\
\end{array}$ & 10 & $\begin{array}{c}0.09 \\
(0.067344)\end{array}$ & $\begin{array}{c}1603.80 \\
(0.544656)\end{array}$ & $\begin{array}{c}0.002 \\
(0.398929)\end{array}$ & $\begin{array}{c}0.01 \\
(0.942286)\end{array}$ & $\begin{array}{c}35.88 \\
(0.019526)^{*}\end{array}$ & $\begin{array}{c}0.08 \\
(0.278879)\end{array}$ & $\begin{array}{c}2600.73 \\
(0.005329) * *\end{array}$ & $\begin{array}{c}620.56 \\
(0.025712)^{*}\end{array}$ & $\begin{array}{c}8797.28 \\
(0.546932)\end{array}$ \\
\hline $\begin{array}{l}\text { Treatments } \\
\text { (Adj.) }\end{array}$ & 95 & $\begin{array}{c}0.13 \\
(0.002694)^{* *}\end{array}$ & $\begin{array}{c}6366.74 \\
(0.000958)^{* *}\end{array}$ & $\begin{array}{c}0.005 \\
(0.012889)^{*}\end{array}$ & $\begin{array}{c}0.01 \\
(0.862295)\end{array}$ & $\begin{array}{c}45.66 \\
(0.000745)^{* *}\end{array}$ & $\begin{array}{c}0.14 \\
(0.017202)^{*}\end{array}$ & $\begin{array}{c}5694.22 \\
(0.000010)^{* *}\end{array}$ & $\begin{array}{c}167.71 \\
(0.828459)\end{array}$ & $\begin{array}{c}56227.46 \\
(0.000025)^{* *}\end{array}$ \\
\hline Error & 20 & 0.04 & 1767.72 & 0.002 & 0.01 & 12.25 & 0.06 & 684.08 & 225.16 & 9727.87 \\
\hline \multicolumn{11}{|c|}{ Contrast Analysis } \\
\hline \multirow{3}{*}{$\begin{array}{l}\text { (i)Among } \\
\text { control } \\
\text { (ii) Among } \\
\text { test } \\
\text { genotypes } \\
\text { (iii) Test-vs } \\
\text { control }\end{array}$} & 2 & $\begin{array}{c}0.59 \\
(0.000170)^{* *}\end{array}$ & $\begin{array}{c}1699.49 \\
(0.399336)\end{array}$ & $\begin{array}{c}0.002 \\
(0.339969)\end{array}$ & $\begin{array}{c}0.01 \\
(0.690446)\end{array}$ & $\begin{array}{c}16.90 \\
(0.274409)\end{array}$ & $\begin{array}{c}0.01 \\
(0.852351)\end{array}$ & $\begin{array}{c}7441.55 \\
(0.000635) * *\end{array}$ & $\begin{array}{c}194.03 \\
(0.437529)\end{array}$ & $\begin{array}{c}8622.04 \\
(0.427748)\end{array}$ \\
\hline & 92 & $\begin{array}{c}0.12 \\
(0.005129)^{* *}\end{array}$ & $\begin{array}{c}5695.39 \\
(0.002124)^{* *}\end{array}$ & $\begin{array}{c}0.005 \\
(0.012395)^{*}\end{array}$ & $\begin{array}{c}0.01 \\
(0.847481)\end{array}$ & $\begin{array}{c}43.76 \\
(0.001027)^{* *}\end{array}$ & $\begin{array}{c}0.14 \\
(0.014734)^{*}\end{array}$ & $\begin{array}{c}5246.77 \\
(0.000010)^{* *}\end{array}$ & $\begin{array}{c}168.91 \\
(0.821943)\end{array}$ & $\begin{array}{c}50403.66 \\
(0.000061)^{* *}\end{array}$ \\
\hline & 1 & $\begin{array}{c}0.38 \\
(0.007355)^{* *}\end{array}$ & $\begin{array}{c}79943.36 \\
(0.000010)^{* *}\end{array}$ & $\begin{array}{c}0.007 \\
(0.089089)\end{array}$ & $\begin{array}{c}0.01 \\
(0.857798)\end{array}$ & $\begin{array}{c}265.98 \\
(0.000151)^{* *}\end{array}$ & $\begin{array}{c}0.03 \\
(0.513141)\end{array}$ & $\begin{array}{c}46711.55 \\
(0.000010)^{* *}\end{array}$ & $\begin{array}{c}1.52 \\
(0.935253)\end{array}$ & $\begin{array}{c}707440.72 \\
(0.000010)^{* *}\end{array}$ \\
\hline
\end{tabular}

\begin{tabular}{|c|c|c|c|c|c|c|c|c|c|}
\hline Source & dff & FNPP & GNPP & PH & FLL & FLW & $\mathbf{L L}$ & LW & SG \\
\hline Block(Adj.) & 10 & $\begin{array}{c}2482.25 \\
(0.025712)^{*}\end{array}$ & $\begin{array}{c}2482.25 \\
(0.025712)^{*}\end{array}$ & $\begin{array}{c}90.29 \\
(0.290880)\end{array}$ & $\begin{array}{c}39874.80 \\
(0.019527)^{*}\end{array}$ & $\begin{array}{c}70890.46 \\
(0.019519)^{*}\end{array}$ & $\begin{array}{c}13.95 \\
(0.612605)\end{array}$ & $\begin{array}{c}0.02 \\
(0.896346)\end{array}$ & $\begin{array}{c}1.27 \\
(0.428152)\end{array}$ \\
\hline $\begin{array}{l}\text { Treatments } \\
\text { (Adj.) }\end{array}$ & 95 & $\begin{array}{c}949.91 \\
(0.470122)\end{array}$ & $\begin{array}{c}949.91 \\
(0.470122)\end{array}$ & $\begin{array}{c}78.83 \\
(0.382095)\end{array}$ & $\begin{array}{c}50743.02 \\
(0.000745)^{* *}\end{array}$ & $\begin{array}{c}90208.87 \\
(0.000745) * *\end{array}$ & $\begin{array}{c}29.13 \\
(0.083577)\end{array}$ & $\begin{array}{c}0.07 \\
(0.045886)^{*}\end{array}$ & $\begin{array}{c}1.13 \\
(0.589938)\end{array}$ \\
\hline Error & 20 & 900.65 & 900.65 & 68.98 & 13608.79 & 24191.83 & 16.97 & 0.04 & 1.19 \\
\hline \multicolumn{10}{|c|}{ Contrast Analysis } \\
\hline $\begin{array}{l}\text { (i) Among } \\
\text { control }\end{array}$ & 2 & $\begin{array}{c}776.12 \\
(0.437529)\end{array}$ & $\begin{array}{c}776.13 \\
(0.437529)\end{array}$ & $\begin{array}{c}119.09 \\
(0.203410)\end{array}$ & $\begin{array}{c}18787.88 \\
(0.274385)\end{array}$ & $\begin{array}{c}33404.70 \\
(0.274323)\end{array}$ & $\begin{array}{c}32.19 \\
(0.175978)\end{array}$ & $\begin{array}{c}0.01 \\
(0.896079)\end{array}$ & $\begin{array}{c}0.75 \\
(0.539710)\end{array}$ \\
\hline \multirow{2}{*}{$\begin{array}{l}\text { (ii) Among test } \\
\text { genotypes }\end{array}$} & 92 & $\begin{array}{c}963.27 \\
(0.454313)\end{array}$ & $\begin{array}{c}963.27 \\
(0.454313)\end{array}$ & $\begin{array}{c}77.77 \\
(0.396640)\end{array}$ & $\begin{array}{c}48622.82 \\
(0.001027)^{* *}\end{array}$ & $\begin{array}{c}86439.48 \\
(0.001027)^{* *}\end{array}$ & $\begin{array}{c}29.36 \\
(0.080961)\end{array}$ & $\begin{array}{c}0.07 \\
(0.046239) *\end{array}$ & $\begin{array}{c}1.01 \\
(0.705962)\end{array}$ \\
\hline & 1 & $\begin{array}{c}34.79 \\
(0.846172)\end{array}$ & $\begin{array}{c}34.79 \\
(0.846172)\end{array}$ & $\begin{array}{c}105.46 \\
(0.230614)\end{array}$ & $\begin{array}{c}295471.04 \\
(0.000151)^{* *}\end{array}$ & $\begin{array}{c}525286.81 \\
(0.000151)^{* *}\end{array}$ & $\begin{array}{c}2.17 \\
(0.724118)\end{array}$ & $\begin{array}{c}0.19 \\
(0.034825)^{*}\end{array}$ & $\begin{array}{c}12.59 \\
(0.003991)^{* *}\end{array}$ \\
\hline
\end{tabular}

$(* \mathrm{P}<=0.05 ; * * \mathrm{P}<=0.01$ Figures in parentheses indicate the $\mathrm{P}$ value $)$ 
Table.3 Clustering pattern obtained by analysis for fodder traits

\begin{tabular}{|c|c|c|}
\hline Cluster & No. of genotypes & Genotypes \\
\hline I & 18 & $\begin{array}{l}\text { OL 1542, JHO-2009-3, SKO 16, SKO 117, SKO 12, SKO 321, SKO 314, SKO 320, SKO 315, SKO } 313 \text {, SKO } 312 \text {, } \\
\text { SKO 316, SKO 323, SKO 25, A. sterlis, Guinea oats, OMS 7, JGO }\end{array}$ \\
\hline II & 40 & $\begin{array}{l}\text { OL 1611, OL 1615, OL 1624, OL 1680, OL 1682, OL 1685, OL 1687, OL 1690, OL 1694, OL 1706, OL 1708, OL } \\
\text { 1710, OL 1711, OL 1714, OL 1720, JHO-322, JHO 851, EC 209750, EC 209472, EC 209408, EC 209402, EC } 209585 \text {, } \\
\text { EC 605835, EC 209547, SKO 109, SKO 10, RFO 5-71, OS 376, OS 363, UPO 032, UPO 276, UPO 033, UPO 093, HJ } \\
\text { 8, HJ 114, NDO 603, NDO 609, OS 329, A. vavilioviana, A. maroccana, }\end{array}$ \\
\hline III & 5 & OL 1612, OL 1625, OL 1636, JO 03-95, OS 6 \\
\hline IV & 26 & $\begin{array}{l}\text { OL 10, Kent, OL 1688, OL 1842, OL 1702, OL 1705, OL 1722, JHO-2009-1, JHO-2001-1, JHO-99-2, EC 605839, EC } \\
\text { 605833, OL 125, EC 605829, OS 374, EC 18850, EC 209684, EC 209307, SKO 105, SKO 27, SKO 163, RO 2001-1, } \\
\text { RO 19, JO 03-93, JO 03-307, OS 7, }\end{array}$ \\
\hline $\mathbf{V}$ & 5 & OL 1684, OL 1686, OL 1692, OL 1635, OL 1837 \\
\hline VI & 2 & EC 209616, EC 209675 \\
\hline
\end{tabular}

Table.4 Clustering pattern obtained by analysis for grain traits

\begin{tabular}{|c|c|c|}
\hline Cluster & No. of genotypes & Genotypes \\
\hline I & 15 & $\begin{array}{l}\text { OL 1542, OL 1624, EC } 209684, \text { OL 1686, OL 1685, OL 1842, OL 1702, EC 209402, JGO 3, SKO 16, SKO } 12 \text {, OS } \\
374, \text { HJ 114, OS 329, UPO } 093\end{array}$ \\
\hline II & 7 & OL 1611, JHO 2001-1, OL 1615, OL 1720, OL 1705, OL 1837, SKO 315 \\
\hline III & 51 & $\begin{array}{l}\text { OL 1612, OL 1684, OL 1682, OL 1687, EC 209307, EC 605829, OL 1680, OL 1714, JHO 851, OL 1722, EC } \\
\text { 18850, JHO 2009-1, EC 209547, SKO 163, SKO 105, OS 376, EC 605836, SKO 10, SKO 25, RO 2001-1, EC } \\
\text { 209585, SKO 321, SKO 314, EC 209675, SKO 117, SKO 320, SKO 312, OL 1688, JHO 2009-3, UPO 032, HJ 8, } \\
\text { RFO 5-71, A. vavilioviana, OL 1692, SKO 109, JO 03-95, OL 1708, SKO 316, OS 6, A. sterlis, SKO 323, JO 03- } \\
\text { 93, OS 363, JO 03-307, RO 19, Guinea oats, OMS 7, OL 1694, NDO 609, NDO 603, OS } 7\end{array}$ \\
\hline IV & 19 & $\begin{array}{l}\text { OL 10, Kent, SKO 27, EC 209403, UPO 033, UPO 276, EC 209750, OL 1690, OL 1636, JHO 322, EC 605833, EC } \\
\text { 209616, EC 605839, OL 1625, OL 125, A. maroccana, OL 1710, JHO 99-2, EC } 209472\end{array}$ \\
\hline V & 3 & OL 1705, OL 1711, SKO 313 \\
\hline VI & 1 & OL 1635 \\
\hline
\end{tabular}


Table.5 Clustering pattern obtained for dual purpose

\begin{tabular}{|c|c|c|}
\hline Cluster & No. of genotypes & Genotypes \\
\hline $\mathbf{I}$ & 48 & $\begin{array}{l}\text { OL 1542, OL 1611, OL 1624, OL 1682, OL 1687, OL 1680, EC 18850, EC 209307, OL } \\
\text { 1722, UPO 033, OL 1714, JHO 851, EC 209750, SKO 27, EC 209750, EC 209408, UPO } \\
\text { 276, SKO 316, A. sterlis, OMS 7, SKO 25, RO 19, JO 03-93, NDO 609, RFO 5-71, OL 1685, } \\
\text { UPO 093, EC 605835, SKO 10, JGO 3, SKO 105, OS 329, JO 03-307, OS 363, JO 03-95, } \\
\text { OL 1842, OL 1702, EC 209684, OS 374, EC 209547, OS 376, EC 209585, SKO 12, SKO } \\
\text { 163, RO 2001-1, OL 1837, OL } 1705\end{array}$ \\
\hline II & 31 & $\begin{array}{l}\text { OL 1612, OL 10, OL 1615, OL 1625, Kent, OL 1684, OL 1686, OL 1688, OL 1690, OL } \\
\text { 1692, OL 1694, OL 1706, OL 1708, OL 1710, OL 1711, OL 1720, JHO-99-2, JHO-322, } \\
\text { JHO-2009-3, EC 605839, EC 605833, OL 125, EC 605829, EC 209472, UPO 032, HJ 8, HJ } \\
\text { 114, NDO 603, A. vavilioviana, A. maroccana, Guinea oats }\end{array}$ \\
\hline III & 5 & JHO 2009-1, JHO 2001-1, OL 1636, OS 6, OS 7 \\
\hline IV & 1 & OL 1635 \\
\hline V & 5 & EC 209616, EC 209675, SKO 320, SKO 315, SKO 313 \\
\hline VI & 6 & SKO 16, SKO 117, SKO 321, SKO 314, SKO 312, SKO 323 \\
\hline
\end{tabular}


Table.6 Average inter-cluster distances in 96 oat accessions for fodder

\begin{tabular}{|l|l|l|l|l|l|l|} 
Cluster & I & II & III & IV & V & VI \\
\hline I & 0.00 & 3.33 & 5.67 & 4.41 & 5.87 & 6.78 \\
\hline II & & 0.00 & 4.16 & 2.31 & 3.66 & 7.67 \\
\hline III & & & 0.00 & 3.66 & 4.31 & 9.99 \\
\hline IV & & & & 0.00 & 3.95 & 9.43 \\
\hline V & & & & & 0.00 & 9.61 \\
\hline VI & & & & & & 0.00 \\
\hline
\end{tabular}

Table.7 Average inter-cluster distances in 96 oat accessions for grain

\begin{tabular}{|c|c|c|c|c|c|c|}
\hline Cluster & I & II & III & IV & V & VI \\
\hline I & 0.00 & 4.86 & 2.78 & 3.90 & 6.22 & 9.06 \\
\hline II & & 0.00 & 3.98 & 3.89 & 4.81 & 6.93 \\
\hline III & & & 0.00 & 2.80 & 4.21 & 8.02 \\
\hline IV & & & & 0.00 & 4.68 & 7.13 \\
\hline V & & & & & 0.00 & 8.19 \\
\hline VI & & & & & & 0.00 \\
\hline
\end{tabular}

Table.8 Average inter-cluster distances in 96 oat accessions for dual purpose

\begin{tabular}{|c|c|c|c|c|c|c|}
\hline Cluster & I & II & III & IV & V & VI \\
\hline I & 0.00 & 2.59 & 4.51 & 9.37 & 7.45 & 5.46 \\
\hline II & & 0.00 & 4.77 & 8.37 & 7.30 & 5.91 \\
\hline III & & & 0.00 & 9.01 & 10.18 & 8.75 \\
\hline IV & & & & 0.00 & 11.24 & 11.11 \\
\hline V & & & & & 0.00 & 5.88 \\
\hline VI & & & & & & 0.00 \\
\hline
\end{tabular}

All the 96 accessions were grouped into six clusters. The critical examination of clusters indicated the presence of high level of genetic diversity in the germplasm collection. The clustering pattern of accessions in each of the six clusters is presented in Table 3, 4 and 5 .

Large number of genotypes in a single cluster depicts that these genotypes are more closely related and had less genetic variation among them. It further implies that hybridization programme employing these genotypes inhabiting a common cluster will be of little use programme and diverse clusters are beneficial for hybridization programme in oat improvement.
Identification of diverse and desirable accessions

In addition to grouping of accessions to different clusters, hierarchical clustering based on squared Euclidean distance was also used to identify the diverse and desirable accessions in terms of inter cluster distance.

The inter-cluster distances were larger than the intra-cluster distances indicating wider genetic diversity between genotypes of the clusters with respect to the traits considered. Therefore, combinations with high heterotic response and superior recombinants may be obtained through hybridizations between genotypes across the 
clusters (Murty and Arunachalam, 1996). Low levels of intra-cluster distances were pinpointing of narrow genetic variation within a cluster (Table 6-8).

According to criteria followed by Proceedings of AICRP (FCU, 2015) a dual purpose crop should have higher green fodder yield than best check and seed yield should not be less than $10 \%$ of best check. Following this criteria, the best check is OL 10. The 70 genotypes having higher fodder yield than best check (OL 10) are OS 7, JHO-2001-1, OS 6, OL 1636, OL 1722, JHO-2009-1, SKO 10, JO 03-95, UPO 033, OL 1612, JO 03-307, JHO-322, EC 209684, SKO 27, JHO-99-2, OL 1635, EC 209307, UPO 093, JO 03-93, OS 329, OL 1625, OMS 7, OL 1624, OL 1611, OL 1692, OL 1686, SKO 314, OL 1688, OL 1705, OL 1720, EC 605835, RFO 571, OL 1702, JHO-2009-3, OL 1690, JHO 851, OL 1615, OL 1684, OL 1687, OL 1706, JGO 3, OL 1685, EC 18850, OL 1842, A. vavilioviana, EC 605829, OL 1714, OL 1694, OS 363, EC 605833, SKO 320, SKO 105, NDO 609, OL 1682, UPO 032, OL 1837, OL 1680, EC 605839, RO 19, HJ 114, SKO 16, HJ 8, SKO 316, SKO 109, EC 209585, UPO 276, EC 209547, OS 376, SKO 163, RO 2001-1 and these are the best 20 genotypes for grain yield are UPO 093, OL 1611, JHO-2001-1, HJ 114, OS 374, OL 1542, A. maroccana, JHO 851, OL 1635, OS 329, SKO 27, HJ 8, OS 363, EC 209408, EC 209402, OL 1714, OL 1685, OS 376, EC 605833, JHO-2009-1.

A total of 14 genotypes viz; UPO 093, OL 1611, JHO-2001-1, HJ 114, JHO 851, OL 1635, OS 329, SKO 27, HJ 8, OS 363, OL 1714, OS 376, EC 605833, JHO-2009-1 were adjudged superior after evaluating the accessions by following this criterion.

Some of these genotypes fell in different clusters and some lied in same the clusters for fodder, grain and dual purpose. For fodder, OL 1611, JHO 851, OS 363, UPO 093, HJ 8, HJ (114, OS 329 fell in cluster II, implies that they have less genetic differences. Similarly, JHO2009-1, JHO-2001-1, EC 605833, SKO 27 fell in cluster IV and OL 1635 in cluster V. It implies that genotypes in cluster II, IV and V have genetic differences. The best check OL 10 fell in cluster IV. Similarly, for grain purpose HJ 114, OS 329, UPO 093 lied in cluster I, HJ 114, OS 329, UPO 093 fell in cluster II, OL 1714, JHO 851, JHO 2009-1, OS 376, HJ 8, OS 363 fell in cluster III, SKO 27, EC 605833 in cluster IV and OL 1635 in cluster VI. The best check OL 10 lies in cluster IV. For dual purpose, OL 1611, OL 1714, JHO 851, SKO 27, UPO 093, OS 329, OS 363, OS 376 fell in cluster I, EC 605833, HJ 8, HJ 114 fell in cluster II, JHO 2009-1, JHO 2001-1 in cluster III and OL 1635 in cluster IV and OL 10 fell in cluster II. As beta-glucan is very important quality trait, these are the 11 genotypes having higher beta-glucan content than best check (OL 10): OS 7, NDO 603, NDO 609, OS 374, HJ 114, OS 6, OL 1611, OL 1636, OL 1706, RO 19, OS 329, HJ 114 and OS 29 were also evaluated as best dual purpose lines.

Agronomic traits and morphological analysis has been repeatedly used when characterizing large data sets of Avena taxa in gene banks (Diederichsen, 2008, 2009), or smaller sets of oat species, cultivars and landraces (Souza and Sorrells 1991; Sheikhehpour et al., 2014; Boczkowska et al., 2014). As a result, morphological description has become a valuable source of information for breeding and agronomic research programs (Boczkowska et $a l ., 2014)$. Even though morphological traits are generally employed in order to estimate genetic variation since their measurements are not laborious, still, diversity estimation based on morphology alone has limitations. Unfortunately, traits are heavily influenced by the environment, are limited in number and possibly unintentional selection for traits with agronomical value (Nikoloudakis et al., 2016).

So convincingly in the present study, lot of variation has been observed for most of the traits under consideration; therefore, many of these accessions may be included in a core group constituted for association mapping of important traits for dual oat improvement. 


\section{References}

Barbara, F.R., Thomas, AR and Brian, L.J. 1972. Statistic Package, Pennsylvania State University.

Boczkowska, M., and Tarczyk, E. 2013. Genetic diversity among Polish landraces of common oat (Avena sativa L.). Genet. Resour. Crop Evol., 60: 2157-69.

Boczkowska, M., Nowosielski, J., Nowosielska, D., Podyma, W. 2014. Assessing genetic diversity in 23 early Polish oat cultivars based on molecular and morphological studies. Genet. Resour. Crop Evol., 61:927-941.

Clemens, R., and Klinken, B.J.W. 2014. Oats, more than just a whole grain: an introduction. Br. J. Nutr., 112: 1-3.

Diederichsen, A. 2008. Assessments of genetic diversity within a world collection of cultivated hexaploid oat (Avena sativa L.) based on qualitative morphological characters. Genet. Resour. Crop Evol., 55:419-440.

Diederichsen, A. 2009. Duplication assessments in Nordic Avena sativa accessions at the Canadian national gene bank. Genet. Resour. Crop Evol., 56:587-597.

F.A.O. 2012. Production statistics; Food and Agriculture Organisation, Rome.

F.C.U. 2015. Proceedings of AICRP: Fodder crops.

Federer, W.T. 1956. Augmented designs $\left(2^{\text {nd }}\right.$ Ed), Pp. 208. Hawaiian Planters' Record.

Hintum, V., Th, J, L., and Knupffer, H. 1995. Duplication within and between germplasm collections. I Identifying duplication on the basis of passport data. Genet. Resour. Crop Evol., 42: 127-33.
Krull, C.F., and Borlaug, N.E., 1970. The utilization of collections in plant breeding and production. In O. H. Frankel and E. Bennett, ed, Genetic Resour in Plants Their Exploration and Conservation, Davis, Philadelphia, PA, pp. 427-39.

Murthy, B.R., and Arunachalam V (1986) The nature of divergence in relation to breeding system in some crop plants. Indian J. Genet. Pl. Breed., 26:188-98.

Nikoloudakis, N., Bladenopoulos, K., and Katsiotis, A. 2016. Structural patterns and genetic diversity among oat (Avena) landraces assessed by microsatellite markers and morphological analysis. Genet. Resour. Crop Evol., 63:801-811.

Oliver, R.E., Obert, D.E., Hu, G., Bonman, J.M., and Jackson, E.W. 2010. Development of oat-based markers from barley and wheat microsatellites. Genome 6:458-71.

Rahore, A., Parsad, R., and Gupta, V.K. 2004. Computer aided construction and analysis of augmented designs. J. Ind. Soc. Agri. Stat., 57: 320.

Rasane, P., Jha, A., Sabikhi, L., Kumar, A., and Unnikrishnan, V.S. 2015. Nutritional advantages of oats and opportunities for its processing as value added foods. $J$. Food Sci. Technol., 52: 662-75.

Sheikhehpour, S., Bahraminejad, S., and Cheghamirza, K. 2014. Morphological and molecular genetic variations of oat genotypes grown in Kermanshah, Iran. Mol. Biol. Res., 41:4023-4030.

Souza, E., and Sorrells, M.E. 1991. Relationships among 70 North American oat germplasm: I. Cluster analysis using quantitative characters. Crop Sci., 31:599-605.

\section{How to cite this article:}

Rajvir Kaur, Rahul Kapoor, Yogesh Vikal and Kamaljit Kaur. 2018. Assessing Genetic Diversity in Dual Purpose Oat (Avena sativa L.) Cultivars Based on Morphological and Quality Traits. Int.J.Curr.Microbiol.App.Sci. 7(05): 1574-1586.

doi: https://doi.org/10.20546/ijcmas.2018.705.187 